\title{
High-Performance Computing and Engineering Applications in Electromagnetics
}

\author{
Ning Yuan, ${ }^{1}$ Joshua Le-Wei $\mathrm{Li}^{2}{ }^{2}$ Jun $\mathrm{Hu}^{,}$and Ashutosh Bhardwaj ${ }^{3}$ \\ ${ }^{1}$ Department of Electrical and Computer Engineering, University of Houston, Houston, TX 77004, USA \\ ${ }^{2}$ Department of Microwave Engineering, University of Electronic Science and Technology of China, Chengdu 610054, China \\ ${ }^{3}$ Department of Physics \& Astrophysics, University of Delhi, Delhi 11007, India
}

Correspondence should be addressed to Ning Yuan, nyuan@uh.edu

Received 19 December 2011; Accepted 19 December 2011

Copyright (C) 2012 Ning Yuan et al. This is an open access article distributed under the Creative Commons Attribution License, which permits unrestricted use, distribution, and reproduction in any medium, provided the original work is properly cited.

With continuously enhanced computing power and practical application demand, high performance computing and its engineering applications in computational electromagnetics turn to be a topic of great importance and extensive interests. In recent years, some significant progresses have been achieved in computational efficiency enhancement by (a) developing new numerical techniques, (b) improving existing electromagnetic numerical methods (in terms of, e.g., unstructured mesh, faster direct or iterative solutions, higher-order basis functions, and characteristic basis functions), and (c) implementing parallel programming techniques such as message passing interface (MPI), OpenMP or graphics processing unit (GPU) technique. There is, however, still a large margin beyond it to move. In this special issue on high-performance computing and engineering application in electromagnetics, we have solicited 12 papers on some recent advances in the field covering a wide range from new techniques to applications of developed techniques.

The paper by $\mathrm{H}$. Guo et al. of this special issue proposes a multilevel-SAI preconditioner to accelerate the convergence rate of Krylov iterations for solving the matrix equation of the method of moments (MoM) based on the hierarchical matrix method. The numerical performance of the preconditioner is investigated in detail and proved to be more efficient than the conventional "single level" preconditioners. The paper by Y. Yan et al. describes the Message Passing Interface (MPI) parallel implementation of the MoM solution with higher-order basis functions. The method can be used to calculate the radar cross section (RCS) of various electrically large objects with high efficiencies. The paper by J. Gómez et al. presents an efficient EM solver based on the MoM with the inclusion of hybrid (asymptotic and rigorous) techniques, MPI, multilevel fast multipole algorithm (MLFMA), and domain decomposition methods. The EM solver is used to the design and optimization of an EBG antenna, and gain enhancement was achieved by using the EBG structure.

The paper by X. L. Travassos et al. of this special issue presents optimization problem formulations to design meander line antennas for passive UHF radiofrequency identification tags based on given specifications of input impedance, frequency range, and geometric constraints. The paper by Z. Zhang et al. applies OpenMP to parallelize the simulation of triaxial induction logging tools in 1D multilayered anisotropic formations based on the transmission theory. The parallel process is explained in detail, and the effect of the parallel code is demonstrated.

The paper by M.-L. Yang and X.-Q. Sheng applies the finite element-tearing and interconnecting (FETI) method with the absorbing boundary condition $(\mathrm{ABC})$ to characterize electromagnetic scattering by large inhomogeneous objects. Two algorithms of FETI method are implemented, and their performances are investigated in detail. The paper by Z. X. Huang et al. proposes an optimized operator splitting methods for numerical integration of the timedomain Maxwell's equations. It is found that the optimized schemes with coarse discrete grid and large CFL number can obtain satisfactory numerical results. The paper by W. Chao and $\mathrm{H}$. Li is a review of EM-scattering methods for two general cases of anisotropic spheres, that is, Cartesian anisotropic and spherical anisotropic. The paper uses plane wave decomposition and $\mathrm{T}$ matrix to deal with Cartesian 
anisotropic sphere and separation of variables for spherically anisotropic sphere.

Other 4 papers all focus on the FDTD method. The paper by Z. L. He et al. presents a parallel finite difference time domain (FDTD) method to analyze electrically large targets on a super computer. The influence of different virtual topology schemes on parallel performance of parallel FDTD is studied in detail, and general rules to obtain the highest efficiency are presented. The paper by K. Narahara developed an FDTD model of the field-effect transistors (FETs) by using piecewise-linear modeling to eliminate route-finding routines in an FDTD solver. Then FETs are characterized in FDTD scheme without significant computational costs for analysis of traveling-wave field effect transistors (TWFETs). The paper by W. Bing et al. describes a TDIE/FDTD hybrid method for calculating the transient responses of thin wire above a lossy half space. The method is highly efficient and requires less memory. The paper by D.-C. Chang et al. of this special issue introduces a hardware acceleration technique for the parallel finite difference time domain (FDTD) method using the SSE (streaming SIMD (single instruction multiple data) extensions) instruction set. The implementation of SSE instruction significantly improves the performance of the parallel FDTD.

Ning Yuan

Joshua Le-Wei Li

Jun $\mathrm{Hu}$

Ashutosh Bhardwaj 

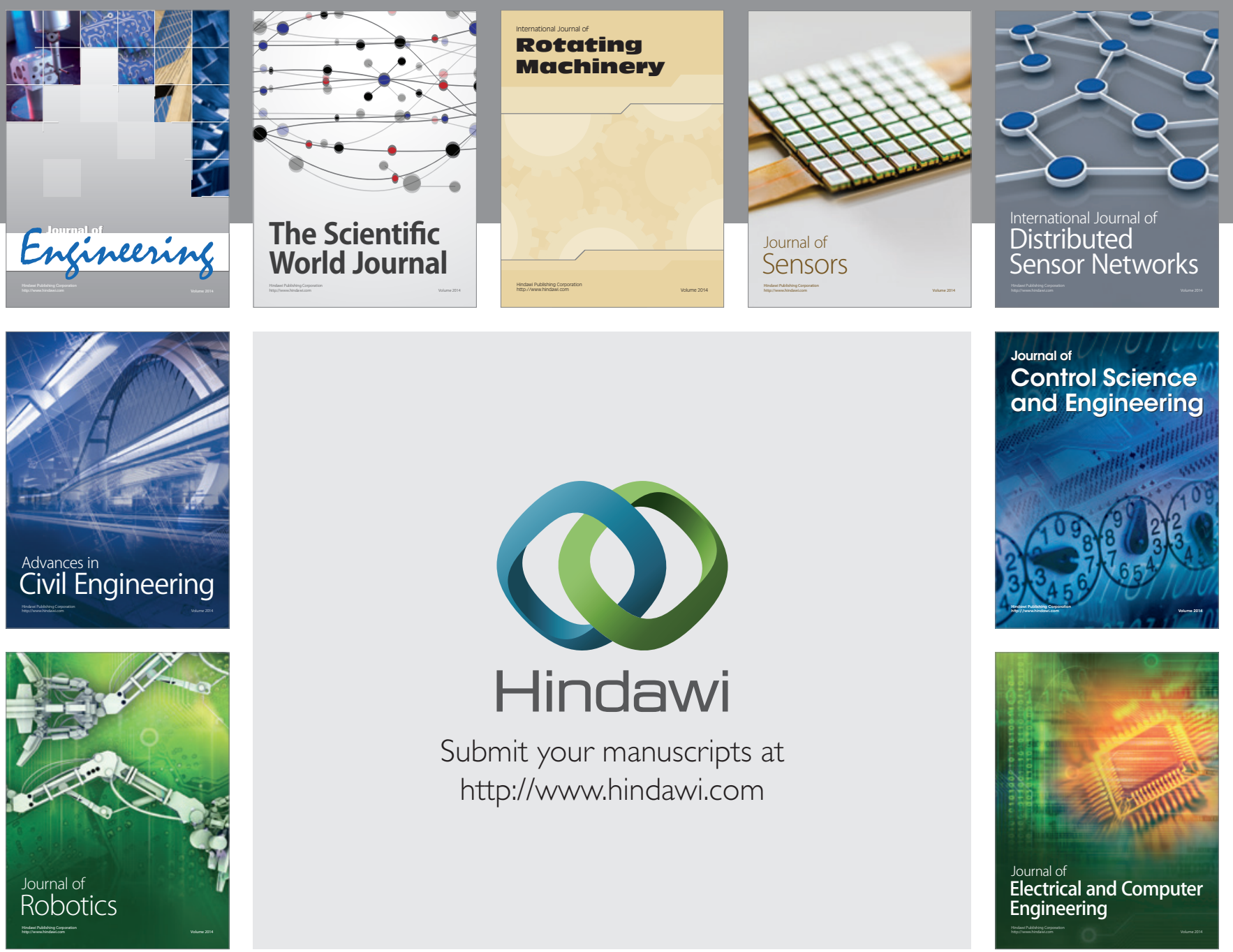

Submit your manuscripts at

http://www.hindawi.com
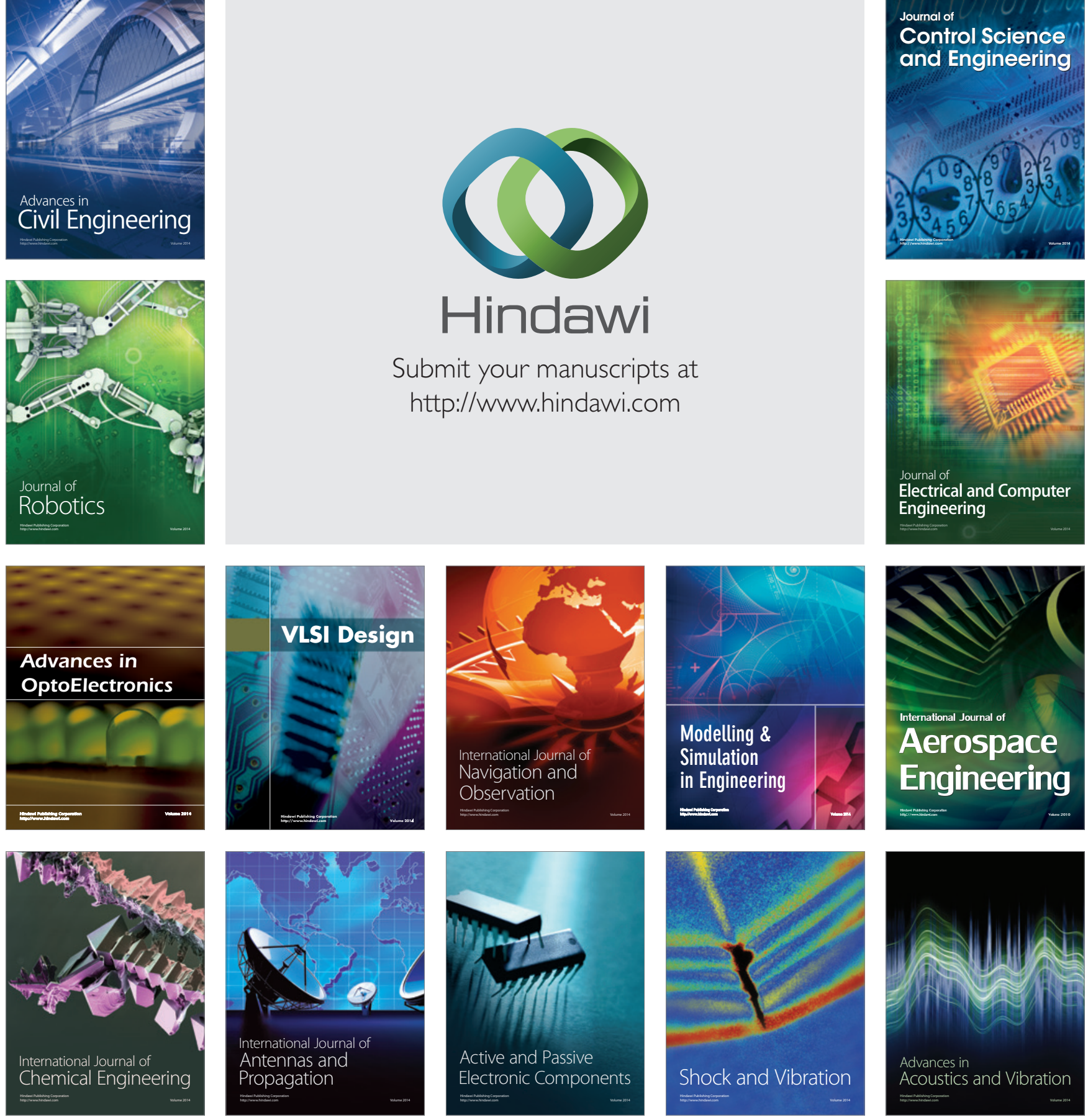